\title{
Investigación narrativa en perspectiva crítica: reflexión metodológica
}

Primer semestre de 2022 - pp. 89-100 Segunda época

N. 0

Narrative Investigation Pesquisa narrativa em in Critical Perspective: perspectiva crítica:

A Methodological Reflexão metodológica

Reflection
Ricardo Antonio Castaño Gaviria* https://orcid.org/0000-0001-9348-6406

Giseh Solans Guisao Gil ${ }^{* *}$ https://orcid.org/0000-0002-6708-3555
Para citar este artículo

Castaño-Gaviria, R. A. y Guisao-Gil, G. S. (2022). Investigación narrativa en perspectiva crítica: reflexión metodológica. Folios, (55). https://doi.org/10.17227/folios.55-12344

* Posdoctor en Educación, doctor en Educación y Contemporaneidad. Docente Universidad de Antioquia.

Correo: ricardo.castano@udea.edu.co

** Magíster en Educación. Docente Universidad de Antioquia.

Correo: giseh.guisao@udea.edu.co 


\title{
Resumen
}

Este artículo surge de la investigación Experiencias descentralizadas de formación docente desde un enfoque biográfico-narrativo, llevado a cabo en la Normal Superior del Municipio de Abejorral, Antioquia, durante el año 2019, la cual tuvo por objetivo conocer los efectos en la formación docente desde experiencias descentralizadas, a partir de la investigación narrativa, en términos de las trayectorias e identidades profesionales. Metodológicamente la investigación se desarrolla desde un enfoque biográfico narrativo reflexivo, el cual contempla la escritura y la construcción del relato en una continua crítica, reflexión y discusión sobre la formación del sujeto. Así, proponemos trabajar la narrativa a la luz de experiencias comunes, llevando la producción narrativa a un momento de problematización. La investigación constituye una ruta crítica para pensar la formación, donde puedan emerger características sociales e históricas de la producción de la identidad profesional. Dentro de las conclusiones se evidencia, que escribir "solo" o desde la individualidad, no permite la reflexividad frente al proceso escritural, por el contrario, el acompañamiento aporta a la perspectiva de un trabajo donde el otro se vuelve un espejo que aporta a la problematización y a la toma de postura política de la propia trayectoria.

\section{Palabras clave}

enfoque narrativo; formación docente; maestro rural; identidad profesional; reflexividad

\begin{abstract}
This article arises from the investigation Decentralized Experiences of Teacher Training from a Biographical-Narrative Approach, carried out in the Normal Superior of the Municipality of Abejorral Antioquia during 2019, which aimed to know the effects on teacher training from decentralized experiences, based on narrative research, in terms of trajectories and professional identities. Methodologically, this text is developed from a reflective narrative biographical approach, which contemplates the writing and construction of the story in a continuous criticism, reflection, and discussion on the formation of the subject. Thus, we propose to work the narrative in the light of experiences, taking the narrative production to a moment of problematization. Research constitutes a critical path to think about training, where social and historical characteristics of the creation of professional identity can emerge. Within the conclusions it is evident that writing "alone"; or from the individuality, does not allow reflection in front of the writing process, on the contrary, the accompaniment contributes to the perspective of a work where the other becomes a mirror that contributes to the problematization and the taking of political position of the own trajectory.
\end{abstract}

\section{Keywords}

narrative approach; teacher training; rural teacher; professional identity; reflexivity

\section{Resumo}

Este artigo surge da pesquisa Experiências descentralizadas de formação docente a partir de um enfoque biográfico-narrativo, realizada na Normal Superior do Município de Abejorral Antioquia em 2019, a qual teve como objetivo conhecer os efeitos na formação de professores a partir de experiências descentralizadas, a partir da pesquisa narrativa em termos das trajetórias e identidades profissionais. Metodologicamente, a pesquisa se desenvolve a partir de uma abordagem biográfica narrativa reflexiva, que contempla a escrita e a construção do relato em uma contínua crítica, reflexão e discussão sobre a formação do sujeito. Assim, propomos trabalhar a narrativa à luz de experiências comuns, levando a produção narrativa a um momento de problematização. A pesquisa constitui um caminho crítico para pensar a formação, onde podem emergir características sociais e históricas da produção da identidade profissional. Dentro das conclusões fica evidente que a escrita "sozinha" ou a partir da individualidade, não permite a reflexividade diante do processo escritural, pelo contrário, o acompanhamento contribui à perspectiva de um trabalho onde o outro se torna um espelho que contribui para a problematização e à tomada de posição política da própria trajetória.

\section{Palavras chave}

abordagem narrativa; formação de professores; professor rural; identidade profissional; reflexividade 


\section{Introducción}

En este texto presentamos la sistematización y reflexión metodológica de la experiencia investigativa desarrollada en el marco del proyecto de investigación denominado "Experiencias descentralizadas de formación docente desde un enfoque biográfico-narrativo", llevado a cabo en la Normal Superior del municipio de Abejorral (Antioquia) durante el 2019.

Como referencia de entrada, debemos reconocer que el campo de investigación narrativa ha terminado constituyéndose como un ejercicio de indagación. Este concepto fue trabajado por John Dewey en 1910, y en 1996 el Consejo Nacional de Investigación de Estados Unidos de América (Reyes y Padilla, 2012) lo presentó como una forma de la que tanto científicos como estudiantes se apropian para comprender y conocer el mundo cotidiano, o sus actividades en investigaciones de campo.

Así, la indagación se ha utilizado en muchas disciplinas e implementado como recurso investigativo; sin embargo, para hablar de esto en educación, es necesario generar una reflexión crítica frente a su estructura y procedimiento metodológico. Se retoma la investigación narrativa desde la concepción de contar con libertad lo vivido, con la intención de reconstruir memorias individuales y colectivas. Se adopta esta manera de contar como una confesión cotidiana, con la cual se habla de modo casual e informal (Valles, 1999).

La conversación que cada sujeto entabla, aunque devela asuntos desde la percepción subjetiva que se ha formado, también permite entablar cuestionamientos desde lo narrativo, a la luz de problemas y dilemas de contexto, los cuales constituyen discursos y prescripciones de la formación del maestro de hoy. Es en este sentido que se considera que la investigación biográfico-narrativa tendría que plantearse en momentos de reflexión, tematización y crítica para desarrollar el trabajo de campo.

La perspectiva metodológica aquí propuesta se proyecta desde un enfoque biográfico-narrativo reflexivo, que contempla la escritura crítica entendida desde la construcción del relato en una continua reflexión y discusión acerca de la formación del sujeto.

Con la escritura autobiográfica realizada se pretende, en primer lugar, evidenciar los aspectos constitutivos desde una mirada de sí mismo. Luego, se busca contrastar estos relatos desde la lectura en voz alta, para que en el grupo se identifiquen posibles experiencias comunes o se amplíe el espectro de lo que viven otros, aun estando en situaciones similares. 
Después de estos momentos de socialización se pasa a una producción narrativa, a un momento de problematización. Esta se constituye en la ruta crítica donde puedan emerger características sociales e históricas de la producción de la identidad profesional del maestro rural. En este caso se plantean tres momentos de indagación narrativa desde los cuales se invita a los sujetos a comprender críticamente los elementos que configuran la posibilidad del relato en el desarrollo de una trayectoria de formación.

Desde esta orientación metodológica se convoca a los maestros a abrir los ojos y oídos a otras realidades, mirándose a sí mismo desde otros y comprendiendo a los otros desde sí mismos. Para este giro, es necesaria una escucha intensa en cada encuentro, hablar de sí pretendiendo identificar las trayectorias individuales y colectivas que forman parte de nuestro quehacer profesional. Desde este aspecto se retoma a Delory-Momberger (2014) para nombrar lo significativo que es trabajar para reconocerse a sí mismo desde el reconocimiento de los otros, a lo cual también se le llama biografiarse.

La participación de los sujetos en la expresión y construcción de sus relatos los ubica en reflexiones sobre su acontecer, con el objeto de apropiarse de su experiencia, de unos temas y saberes implícitos. Sin embargo, es importante precisar que esta postura metodológica no se debe confundir con la idea de una investigación narrativa que ofrece cura o solución definitiva a las encrucijadas propias del campo profesional. Como alternativa, se busca evidenciar la capacidad de reelaborar a partir del trabajo biográfico-narrativo una conciencia de su propia trayectoria, desde una condición crítica-relacional con el contexto y con las experiencias que otros han vivido.

\section{Momentos de la indagación narrativa}

Se considera necesario en esta aproximación metodológica constituir un "camino", una forma de abordaje que permita superar la literalidad del relato y que se evidencien el sujeto y la cultura frente a las contradicciones y tensiones que se les presentan desde lo positivo o negativo que los constituye (Matos et al., 2018). Se plantea esta propuesta metodológica como una alternativa que busca conocer lo que viven los sujetos para hacer una construcción individual que a la par permita identificar una memoria colectiva, que subyace en una trayectoria de formación y que comparte algunas características.

A continuación, se presentan tres momentos de esta propuesta: memoria y relato, tematización del relato y problematización del relato y el procedimiento realizado en el campo investigativo.

\section{El tratamiento de la memoria y el relato}

Se convoca a la lectura en voz alta para escuchar de otras voces que se viven situaciones similares y disímiles, así se esté en contextos con características comunes. De igual forma, se evidencia que los relatos no se construyen en solitario ni son el reflejo de una voz lineal, sino que se hacen en una espiral polivocal, producto de las relaciones y las comprensiones, lo que en últimas se instaura como la intersubjetividad (Arias Cardona y Alvarado Salgado, 2015).

La construcción del relato pasa por la resignificación de los acontecimientos, y desde allí por un necesario ejercicio de memoria y selección. Producirlo sin un ejercicio reflexivo no permite obtener resultados transformadores. Se recomienda tomar el relato como un "producto", una vez se hayan realizado procesos de reflexividad frente a algo que ha sucedido; dotando de sentido la resignificación de la experiencia, su apropiación y comprensión. En consecuencia, la memoria no puede entenderse como un espacio cerrado e inmutable; por el contrario, también es un espacio político, ficcional y de reconfiguración de sentidos a la luz de nuevas experiencias y trayectorias. La relación entre memoria y relato es dinámica y creativa en la medida en que el dispositivo de investigación narrativa lo permita y promueva. No se debe olvidar que la memoria no es fidedigna, es interpretación a la luz de un presente, también afectada por situaciones y posiciones políticas. 
Considerar la relación entre memoria y relato como momento de la iniciación a la indagación narrativa es un punto clave para dar lugar a un proceso reflexivo, sin pretender en esta postura inductiva prever todo lo que puede acontecer en el momento en que se decide hablar de sí, del otro y de un "nosotros" como posibilidad.

En el contexto de la investigación que se realizó con maestros en la Normal Superior en el municipio de Abejorral (Antioquia), evidenciamos la importancia de relacionar críticamente memoria y relato, acompañar la conversación como socialización de los escritos biográficos entre los participantes, dar tiempo a la reflexión sobre los aspectos compartidos o distantes en la experiencia, profundizar en las categorías que emergen durante la socialización y problematizarlas, instaurándose como orientación para llegar a un proceso de escritura espontánea rodeada de conversación, escucha y conocimiento.

El proceso de escritura es un momento crucial en la investigación, porque es allí donde los actores hacen escuchar su voz, plasmando su propia trayectoria. Este es un proceso de construcción individual y de acompañamiento. En palabras de Souza:

La investigación (auto)biográfica en el proceso de formación de formadores se vincula a la idea de que es la persona la que forma y se forma a través de la comprensión que elabora del transcurso de su propia vida, permitiendo al sujeto percibirse como actor de su trayectoria de formación. Mediante la investigación (auto)biográfica, el sujeto produce un conocimiento sobre sí, sobre los otros y el cotidiano, el cual se revela a través de la subjetividad, de la singularidad, de las experiencias y de los saberes, al narrar en profundidad. La centralidad del sujeto en el proceso de formación subraya la importancia del abordaje comprensivo y de las apropiaciones de la experiencia vivida, de las relaciones entre subjetividad y narrativa como principios, que concede al sujeto el papel de actor y autor de su propia historia. (2011, p. 45)

Sin embargo, como se mencionó, este proceso no se hace en soledad, sino en acompañamiento de los investigadores a los participantes como estrategia clave en el proceso de escritura. Durante el encuentro y la conversación atenta, nos permiten señalar la encrucijada en la cual la presencia es algo vívido, latente, para lo cual es necesario darle sentido a un "yo" desde una mirada pedagógica, relacional y darle paso a la interpelación (preguntarse), al reconocimiento del otro desde las situaciones propias, abiertos a otras formas de configuración de sentido. El acompañamiento como pedagogía del trabajo biográfico-narrativo implica salir del lugar de la certidumbre de todos los tecnicismos, para ir con el otro al reconocimiento de otros posibles. Este acompañamiento cumple también el papel de identificar la tematización, es decir, lo vinculante que emerge con la construcción del relato.

Considerando que la narración, en palabras de Benjamin (2016), prospera lentamente en el círculo artesanado de la comunicación, no se propone aquí transmitir ni construir los relatos como una información o "reporte", debe surgir el "asunto", la vida del relator para luego recuperar desde allí sus sentidos, tornarse un autor, alguien que se da un propio sentido.

Por esto, en un primer momento la estrategia trabajada con el colectivo de maestros de la Normal de Abejorral fue la conversación intencionada. Los participantes hablaron (memorando) acerca de sus experiencias en torno a la trayectoria de formación descentralizada (rural), intentando no ponerse en lugar individual (singular) sino, a partir de la escucha reflexiva, conectarse en la conversación, que pretende vigorizar la memoria y la comprensión. Para este proceso, el papel del coordinador ${ }^{1}$ es importante y significativo.

Para pensar la relación entre memoria y relato es necesario considerar que la memoria no es algo que está guardado en "algún" lugar, de manera intacta y esperando ser rescatado. En una orientación biográfico-narrativa crítica, la memoria debe ser reconstruida, además de problematizada, como espacio de producción política del sujeto.

1 En este abordaje consideramos que el acompañamiento (pedagógico) es importante para contribuir al cuestionamiento, a poner en evidencia los puntos en común y superar la literalidad del relato. 
Primer encuentro con los maestros de la Normal de Abejorral

Abejorral, donde la gente de antaño se bañaba en la quebrada las Yeguas (dice la creencia popular que esto los hacía inteligentes), es un municipio del oriente antioqueño con un pasado de viajeros y comerciantes de caminos y expansión de la cultura. De importancia histórica por ser ruta y lugar estratégico para la colonia hacia otros territorios, el viejo Caldas; también, como varios municipios de Antioquia, un lugar de disputas y de violencias que marcaron su historia y la de su gente, migrada a varias partes de Colombia. Es en ese escenario donde se inscribe la vida y formación de los maestros y la comunidad educativa de la Normal Superior del municipio.

El primer encuentro pedagógico en Abejorral se dio en junio del 2019. La iniciativa resultó de conversaciones previas entre colegas que reconocimos en la investigación biográfico-narrativa una alternativa para pensar la formación docente en contextos formativos rurales y descentralizados. En esa primera visita, se expresaron las intenciones por parte del equipo de investigadores cooperadores para trabajar en este escenario, se presentaron sus motivaciones, formaciones y actuaciones profesionales. Luego se reconocieron también los asistentes convocados para este ejercicio y sus trayectorias, todo por medio de una estrategia: ser presentado por otro, en este caso por una de las investigadoras que integran este colectivo.

Luego de esa dinámica de expresar lo que conozco del otro, que rompió en parte con las tensiones de un encuentro con desconocidos, se emprendió a hablar formalmente del proyecto de investigación que concentraba la razón de visita al municipio.

Posteriormente, se pasó a un acercamiento conceptual sobre la investigación narrativa como temática central de este primer encuentro. En este espacio, que estuvo a cargo de uno de los investigadores, se desarrollaron temas como la pedagogía negativa (contar para comprender, la necesidad de (de)formar para transformar), entre otros aspectos, como lugar de fundamentación inicial.
Después, se volvió a la postura de continuar con la parte explicativa y formativa del porqué se estaba allí. Se comenzó preguntando a los asistentes qué comprendían por experiencia. Esto dio pie para presentarles a la autora Delory-Momberger, de quien se leyó la siguiente cita:

Para ir más lejos en la comprensión de los niveles de la experiencia, es útil precisar el proceso de construcción de la experiencia misma; dicho de otra manera, la forma en que metabolizamos la experiencia vivida, la inmediata, en experiencia adquirida, y por la que constituimos acumulativamente nuestros recursos experienciales. (2014, p. 699)

Después de esta socialización para conocer qué significaba para los asistentes la experiencia a partir de la conceptualización de Delory-Momberger, se presentó el instrumento de trabajo para la producción crítico-reflexiva de escrituras narrativas, y se invitó a los asistentes a reconstruir las trayectorias de formación docente, problematizando desde los presupuestos que allí se plantean. En la figura 1.1 se caracteriza el primer momento del trabajo realizado.

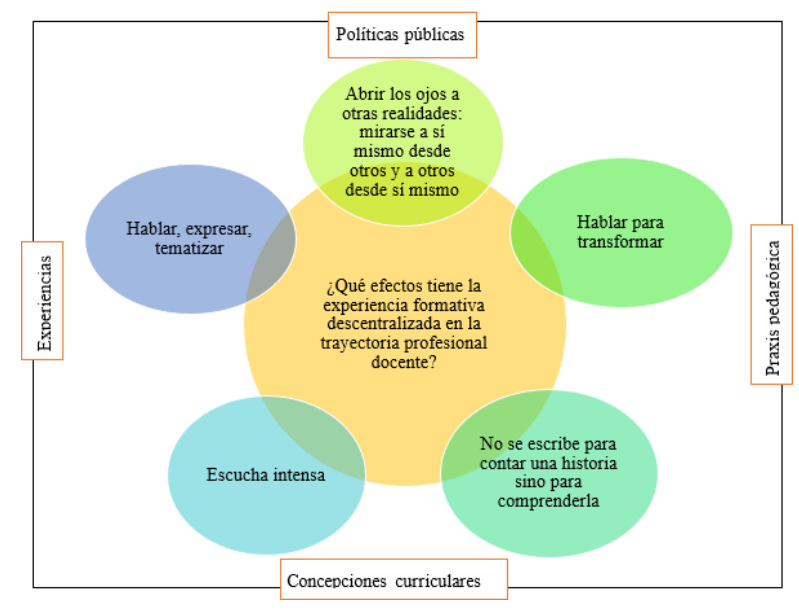

Figura 1. Diagrama para la producción de escritura narrativa en la formación docente

Fuente: elaboración propia.

Luego se convocó a los participantes a escribir un microrrelato a partir de la pregunta ¿Qué efectos tiene la experiencia formativa descentralizada en la trayectoria profesional docente? Apoyados en 
el diagrama realizado por el grupo investigador, se puntualiza que ya se habían socializado entre los participantes algunos conceptos, como que la experiencia formativa era hablar del pregrado, lo descentralizado tenía que ver con su vinculación a una institución universitaria en condiciones de educación a distancia y que la trayectoria profesional se refería a cómo veían su biografía desde la formación hasta el presente. Sobre esto último, es importante precisar que la pregunta se orientó a partir de dos cuestiones: desde los efectos de la formación descentralizada en los sujetos y desde los efectos de esa formación en las trayectorias profesionales. Los escritos se convierten en textos narrativos una vez se cuenta la experiencia particular que cada participante ha vivido.

\section{Elaborar un relato de formación}

Los maestros participantes en el primer encuentro respondieron la pregunta ¿Qué efectos ha tenido la experiencia formativa descentralizada en la trayectoria profesional docente? Para ello, se dispuso de una hora, en la que podían desplazarse a otros lugares; luego, se convocó nuevamente al encuentro y se pusieron en común, a modo de conversación, sus experiencias, El siguiente es uno de los fragmentos significativos de ese ejercicio.

\section{La renuncia}

\section{(Relato)}

Para acceder a la formación profesional docente, se hizo necesario renunciar a un estilo de vida, posesiones, costumbres, comodidades, seguridades. El éxodo que obedecía a un ideal, me marcó como momento difícil, el apego al territorio, dejar mi entorno, mi colegio, la huerta del hogar, las chambranas frente a las cuales veía los amaneceres, la estructura de mi habitación, la generosidad de los espacios, fue un momento de pánico que en principio implicó desarraigo, pero a la vez me causaba curiosidad la posibilidad de emancipación del grupo familiar.
Desde ese momento, empiezo a tejer un compendio, una vivencia que se volvía interesante, la inmensidad de la ciudad, mi nueva residencia, el grupo de compañeros durante el primer semestre de Licenciatura, el campus universitario, las materias pedagógicas como solíamos llamarlas, la asistencia a eventos teatrales, espacios culturales, cuenteros, los conciertos, visitas a museos, sumaron elementos novedosos que describo porque hicieron parte de una configuración de vivencias, las cito porque sin duda alguna me abrieron una panorámica para interpretar la diversidad, constituyendo así experiencias de vida que permearon el ámbito de la formación.

En principio, mi programa de pregrado se dio en modalidad presencial, luego frente a una oportunidad laboral, se hizo necesario continuar en modalidad semipresencial, un proceso descentralizado que empezó a coexistir con mi desempeño docente en mi municipio de origen. El recorrido de cinco semestres en modalidad presencial ofreció elementos valiosos a nivel disciplinar; no obstante, me causaba asombro que la institucionalidad, los diversos contextos educativos, la política educativa pública, requiera de tantas demandas, para las cuales no me sentía preparado y desde las cuales no se profundiza en los claustros universitarios.

Vincularme de lleno a un modelo educativo descentralizado empezó a constituir una etapa significativa en mi proceso formativo, puesto que me daba la posibilidad de realimentarlo con mis prácticas pedagógicas tempranas, justamente en un laboratorio de formación pedagógica: la Escuela Normal Superior de Abejorral, lo cual reafirmó mi gusto por la educación y es justo en ese momento que mi experiencia formativa empezó a revestirse de sentido.

\section{Temas emergentes en los relatos de maestros}

Después de este primer encuentro, luego de leer los relatos de los maestros participantes del taller, los investigadores-colaboradores discutieron, identificaron y realizaron una selección de las temáticas emergentes en las narrativas de los maestros participantes, las cuales se utilizarían como punto de 
partida para el diseño del segundo encuentro con el colectivo de la Normal de Abejorral.

En la revisión general de los escritos que se produjeron en el primer encuentro, se evidenciaron de manera coincidente las siguientes temáticas en las narrativas a la hora de reconstruir sus trayectorias:

- Momentos de socialización y contacto con otros

- Maestro como actor social y actor político

- ¿Construir nuestros relatos desde el desamparo?

- Construir un relato vinculante a la realidad social

- Formación desde el esfuerzo individual y social

- El ser necesita de otros para ser lo que se es

- Pregunta y nuevos problemas

- Experiencias valerosas

- Construcción de la identidad

- Precarización y no vinculación del relato en una experiencia social

- Hábitos profesionales del maestro rural

- Tiempos del maestro

Cuando los investigadores mostraron a los participantes que fueron estos los ejes que permitieron señalar cómo existen unas características comunes en la constitución de sus relatos y subjetividades, aquellos hablaron de cómo veían esas categorías y se pudieron identificar nuevas reflexiones.

\section{Tematización del relato (un momento para pensar en lo dicho)}

El momento que denominamos de tematización dentro de la investigación narrativa se entiende como la capacidad para identificar temas clave que conectan las experiencias de los sujetos que construyen sus relatos; es un momento para que se actualicen esas memorias y narraciones, de manera que se reconstruyan los relatos vinculando experiencias a temas emergentes. Este segundo momento retoma los relatos con sus descripciones literales y trató de buscar conexiones con las experiencias que otros han vivido, ubicando los sujetos en un colectivo, en este caso un espacio de reflexión de maestros con sus trayectorias y experiencias formativas.

La tematización constituye un refuerzo al acompañamiento pedagógico, por cuanto la conversación e identificación de lo común busca el vínculo entre los partícipes, autores y coautores de lo que puede ser relatado. El efecto que se produce al sacar la conversación de lo singular a lo plural es que se puedan reconocer tramas de significados compartidos, puntos no vistos o considerados de manera individual. En el momento que la tematización se propone como parte de la indagación narrativa se va profundizando tanto en contenidos como en formas de abordaje de la producción de los relatos.

La tematización es un momento de vínculo, que potencia capacidades para trabajar a futuro en estos temas, buscando proyectar la voz de la "queja" o "demanda" que ponen los sujetos al contar los momentos de dificultad a la comprensión de las situaciones como algo que acontece.

\section{Descripción metodológica del segundo encuentro con el colectivo de maestros}

Este encuentro se trabajó a partir de una estrategia de conversación activa, escucha intensa y lectura de los contenidos emergentes en los textos que fueron producidos en el primer encuentro. Luego de la socialización y conversación, se dio paso a la complejización de las temáticas encontradas - lo que en la investigación llamamos lectura crítica-. Se creó una red de ideas (tela de araña), donde cada temática tenía un hilo unido al centro que era la pregunta de donde surgieron los textos, y la otra punta podía conectarla un participante a otra temática. Después, en subgrupos, se pasaría a elaborar una construcción escrita a partir de una nueva identificación de los vínculos entre temas y problemas.

La estrategia de trabajo permitió a los participantes escoger una temática previamente evidenciada en los textos, luego se les pidió que realizaran una reflexión individual e interna para presentarla oralmente. Posteriormente, en una rueda tipo reloj, con los doce temas que emergieron de la sesión anterior, cada participante expuso cómo se relaciona 
su temática con otra u otras. Los demás escuchaban atentamente para decidir qué temáticas eran vinculantes con su experiencia y trayectoria personal. ${ }^{2}$

\section{Momentos de socialización de experiencias y reflexión}

Los participantes escogieron una temática de las emergentes de su propio trabajo, las cuales fueron expuestas en la red de ideas. Los investigadores les solicitaron explicar lo que designaban estas temáticas y se procedió a reflexionar de manera individual, luego cada uno expuso oralmente las ideas que vinieron a su mente en ese momento. Los siguientes fragmentos presentan las voces de los maestros participantes tal como fueron expresadas.

\section{Construcción de identidad profesional:}

"Formación, práctica docente, estilo docente, espacios colores, recuerdos acogedores para los procesos de enseñanza. [Puntualiza en la importancia de] identificar unos rasgos identitarios del maestro con formación descentralizada y experiencia en contextos rurales. La identidad como problema de formación". (PJ)

Momentos de socialización, discurso como parte vital del docente

[...] identidad profesional nace desde una familia de maestros, se inspira en el hacer de otros en casa, del contacto con ellos, sus discursos sociales, además las prácticas que observaba y compartía con ellos de encuentro con otros. Un maestro que es "todero", puesto que, en contextos rurales, el docente es la única presencia del Estado y el interlocutor válido para todos los procesos comunitarios y culturales, así se ejerce la docencia en zonas rurales del municipio de Abejorral. Rasgos característicos de este maestro que inspira es la vitalidad, un docente que es reconocido ampliamente y es sujeto público, con convicción y capacidad de decisión. (Px)

\section{Experiencias valerosas}

Entramado de posibilidades desde el ángulo que se mire ser hacer, emociones formación praxis

2 Este párrafo describe lo que en la investigación concebimos como lectura crítica. contexto experiencia vida, rescate del ser y hacer, experiencias transversalizadas por las políticas educativas, social profesional. Todas las experiencias tienen un tiempo. (PS)

\section{Tiempos del maestro}

Tomado como camino para transitar, encuentros, momentos, también depende de la edad, la experiencia acumulada vincula cada vez más, por ello no se deja de ser maestro, es una forma de vida, si bien puede finalizar la vida laboral formal, el ser de maestro se mantiene. (PN)

Formación desde el esfuerzo individual y social

Formación individual social, físico, político, familiar, fundadores. La formación ha sido limitada, ha significado esfuerzos y renuncias personales, se ha asumido desde el esfuerzo personal y familiar para poderlo lograr, para acceder, una vez se accede algunas instituciones como la normal, los programas descentralizados de algunas universidades. Actualmente el Estado ha aportado para la formación profesional docente con programas de maestría. Se han desconocido las necesidades de formación permanente del maestro, los programas se han diseñado por oferta y no por demanda. Las familias han sido bastante afectadas en lo económico y por ausencias del maestro para poderse formar. Muchos actores sociales que han aportado a lo que hemos llegado a ser. Esfuerzos individuales que justifican el beneficio colectivo.

\section{Maestro como actor social}

Es la imagen del maestro rural. [PJP]

¿Construir nuestros relatos desde el desamparo?

Maestros en el filo de la navaja, devaluación de la profesión docente, políticas que sobrepasan la capacidad del maestro. Un elemento que produce desamparo en el maestro es ver un niño sufrir, presenciar la negación de una familia, también cuando el maestro no ama la profesión. En la profesión docente se necesita del reconocimiento del otro para construirse, construirse y reconstruirse maestro.

El conocimiento que genera capacidad y proporciona herramientas para la toma de decisiones, 
esto evitaría ponerle el cuerpo, aunque también puede suceder que ante el conocimiento profundo de las situaciones, la vinculación afectiva provoque mayor vinculación del cuerpo. También es importante acercarse a los planteamientos de la escuela desconcentrada en dos sentidos, uno el de la escuela que ha perdido su concentración en la tarea formativa y dos en una escuela que puede reinventarse en lo local a partir de los planteamientos constitucionales de descentralización, una escuela que está en diferentes lugares, romper el aislamiento de la escuela y acercarla a la comunidad, no obstante es necesario profundizar en la escuela comunidad rural, figura que ha permanecido a lo largo del tiempo en la experiencia propia del municipio de Abejorral. (PO)

Precarización, no vinculación del relato a una experiencia social

Precarización, tanto desde las trayectorias, como en el ejercicio real en el contexto, condiciones que no son las óptimas para el desarrollo de la profesión. Cómo lo precario se convierte en una oportunidad, cómo se lleva la precariedad hacia la identidad y algunas resistencias a llevarse hasta la afectación de la identidad profesional docente, hacia una identidad erosionada. (PF)

El ser necesita de otros para ser el ser que se es...

Profesión humanizante, experiencia y decisión. Lo que yo hago... a quién afecto. Del otro aprendo no solo lo que se debe, sino también lo que no se debe hacer, los otros enseñan, incluso los niños son los mejores maestros. Renovarse cada día, construcción de la vida desde el mundo de la vida. Relato vinculante a la realidad social. Hablar desde el mundo de la vida construido desde diferentes ámbitos. Relación vinculante hace que el ser del maestro se vincule desde lo social, más allá de la función. Imagen de maestro, estilo/modo de ser maestro. (PN)

\section{Construir un relato vinculante desde la realidad} social

El maestro y las políticas públicas: Condiciones básicas. El ejemplo como fuente de inspiración. El maestro que es todo en la comunidad. Un maestro que inspira a otros maestros. Maestros que han convivido con actores del conflicto, vivir con ellos y padecer su intervención. Los esfuerzos físicos que requiere laborar en las veredas, las adiciones de tiempo, una profesión que atraviesa el cuerpo. (PA)

En este sentido, uno de los investigadores anota que la pedagogía también se habita desde la contradicción, pedagogía negativa cuyo dispositivo podría ser la indignación.

\section{Hábitos maestro rural}

Lectura de contexto, liderazgo comunitario y trabajo cooperativo, colaborativo, trabajo entre pares, desarrollo psicomotor, formación política y ética, participación activa en la sociedad civil organizada. (PD)

\section{Problematización del relato}

Luego de la relectura de los relatos por parte de los investigadores se procede a señalar temas emergentes e identificados, que se problematizan a la luz de una lectura crítica que se enfoca en un tiempo-espacio, en una época, en un contexto cultural, económico y político, de los cuales se anticipa que se han emanado directrices y orientaciones de política para la formación de maestros.

Se puede reconocer que hay detrás de la construcción del relato unas fuerzas y tensiones que condicionan tanto la posibilidad de organizar la narración como de comprender otras formas de contar. Ya se ha hablado desde la teoría estructuralista de Bernstein (1990, citado en Graizer y Navas Saurin 2011), en la cual se vinculan los microprocesos como lenguaje, transmisión y pedagogía a las macroformas como cultura, educación y contenidos, con la clase social y las relaciones de poder. El momento de problematización entra a dar un valor explicativo al proceso de indagación, de cómo las reproducciones discursivas dentro de unas prácticas pedagógicas 
que son propias de una época y unas circunstancias políticas pueden dotar de similitudes la formación profesional.

\section{Metodología del tercer encuentro}

Luego del segundo encuentro, a partir de los relatos orales presentados por los participantes, se les asignó el trabajo independiente de dos semanas de una construcción escritural con base en las temáticas ya trabajadas. Debían retomar no solo la trayectoria individual, sino también las intervenciones realizadas por los pares; estos textos se debían enviar de manera digital junto con las devoluciones hechas por los investigadores.

Durante la revisión de estos textos elaborados a partir del segundo encuentro, los investigadores identificaron una necesidad latente en este proceso de escritura: el acompañamiento orientado a la construcción de un texto con la rigurosidad académica que solicita el medio para divulgar los productos de este colectivo, pero sin dejar de lado la idea de un texto cargado de vivencias, de una postura crítica y escrito con un tono narrativo.

Este acompañamiento de manera particular enriquece la perspectiva de un trabajo donde el otro se vuelve un espejo que aporta a la problematización y la toma de postura de la trayectoria propia. Es importante tener presente que los escritos finales, aunque se elaboran desde la individualidad, reflejan devoluciones, discusión, verbalización de lo que otros han dicho, las primeras escrituras propias, lo que en últimas demuestra un tejido de la voz autobiográfica y las voces de los autores de quienes se habla, lo que permite una reflexividad y también un nuevo compromiso para continuar.

\section{Conclusiones}

Esta reflexión metodológica no pretende imponer un orden como una rutina para la indagación narrativa; la combinación y el orden también permiten el diseño de nuevos momentos y formas de proceder. La manera de hacerlo la determinan el contexto, las conversaciones, las configuraciones del grupo; solo se sugiere la importancia del acompañamiento, porque este podrá orientar la dirección y profundidad de los momentos.

Lo que predomina desde esta experiencia es un trabajo que debe ir más allá de la mera enunciación de trayectorias aisladas, sin sentido crítico. Por el contrario, se debe buscar el debate, la problematización, intentando que las dimensiones sociológicas y antropológicas de la experiencia que se narran, y se develaron al confrontar los tiempos, el contexto, y las vivencias, también puedan dar sentido y explicación a las lógicas de formación establecidas. La investigación narrativa no solo debe servir para dar resolución a preguntas individuales.

Aunque escribir solo o desde la individualidad permite unos niveles de reflexión como los nombrados por Perrenoud (2007), Suárez et al. (2004), entre otros autores, en este caso se busca flexibilidad. Además del proceso escritural, se buscó convocar la discusión, la verbalización, volver a lo escrito, y seguir tejiendo la voz del autor con las de los autores de quienes se habla; es por esto que el acompañamiento del investigador y los pares aportan a la perspectiva de un trabajo donde el otro se vuelve un espejo que aporta a la problematización y la toma de postura de la trayectoria propia.

\section{Referencias}

Arias Cardona, A. M. y Alvarado Salgado, S. V. (2015). Investigación narrativa: Apuesta metodológica para la construcción social de conocimientos científicos. Rev. CES Psicol, 8(2), 171-181. http:// www.scielo.org.co/scielo.php?script=sci_arttext\&pi$\mathrm{d}=$ S2011-30802015000200010

Benjamin, W. (2016). El narrador. Editorial Metales Pesados.

Delory-Momberger, C. (2014). Experiencia y formación: Biografización, biograficidad y heterobiografía. Revista Mexicana de Investigacion Educativa, 19(62), 695-710.

Graizer, O. L. y Navas Saurin, A. (2011). El uso de la teoría de Basil Bernstein como metodología de investigación en didáctica y organización escolar. 
Universidad Pedagógica Nacional

Facultad de Humanidades

Revista de Educación, 356, 133-158. http://www. revistaeducacion.educacion.es/re356/re356_06.pdf

Matos, R., Castaño, R. y Souza, E. (2018). Pedagogía de la resistencia: La negación como pieza de (de) formación. Praxis Educativa, 22, 94-111. https://doi. org/E-ISSN: 2313-934X

Perrenoud, P. (2007). Desarrollar la práctica reflexiva en el oficio de enseñar. Graó/Colofón.

Reyes, F. y Padilla, K. (2012). La indagación y la enseñanza de las ciencias. Universidad Nacional Autónoma de México, 23, 415-420. https://doi.org/ ISSNE 1870-8404

Souza, E. (2011). Acompañamiento, mediación biográfica y formación de formadores: dimensiones de investigación-formación. Educación y Pedagogía, 23, 41-56. http://aprendeenlinea.udea.edu.co/revistas/index. $\mathrm{php} /$ revistaeyp/article/view/14008/124\%0A15\%0A

Suárez, D., Ochoa, L. y Dávila, P. (2004). Documentacion narrativa de experiencias pedagogicas, 2(17), 16-31. https://revistas.pedagogica.edu.co/index.php/NYN/ article/view/1228/1225

Valles, M. S. (1999). Técnicas cualitativas de investigación social: Reflexión metodológica y práctica profesional. http://mastor.cl/blog/wp-content/uploads/2011/12/ Tecnicas-Cualitativas-De-Investigacion-SocialValles.pdf 